\title{
Quantum Dynamics of a Nanomagnet driven by Spin-Polarized Current
}

\author{
Yong Wang and L.J. Sham* \\ Center for Advanced Nanoscience, Department of Physics, \\ University of California, San Diego, La Jolla, California 92093-0319, USA
}

\begin{abstract}
A quantum theory of magnetization dynamics of a nanomagnet as a sequence of scatterings of each electron spin with the macrospin state of the magnetization results in each encounter a probability distribution of the magnetization recoil state associated with each outgoing state of the electron. The quantum trajectory of the magnetization contains the average motion tending in the large spin limit to the semi-classical results of spin transfer torque and the fluctuations giving rise to a quantum magnetization noise and an additional noise traceable to the current noise.
\end{abstract}

PACS numbers: 75.75.Jn,72.25.Mk,05.10.Gg

Introduction The spin transfer torque (STT) [1, 2] is both a fundamental process in magnetization dynamics and important to spintronics in memory and information processing integration. The magnetization dynamics driven by spin-polarized current via STT has made great progress by experiments and by a "semiclassical" theory of treating the magnetization dynamics classically and the polarized current transport and the spin waves quantum mechanically (see a series of reviews introduced by Ref. [3]).

Recent development in fast time-resolved measurements [4 6] and coherent control[7] have made possible studies of magnetization dynamics and fluctuations not masked by the inhomogeneity effects of the measurement and the prospect of precision control. These recent experiments including Ref. [8], by showing the stochastic nature of the magnetization dynamics at short time intervals, highlight three important aspects of the subject. First is the stochastic motion which may lead to an understanding of noise, a fundamental issue in magnetization dynamics [9]. Second, in interconnected systems at the nanoscale, the particulate nature of the current electrons imprints shot noise on the magnetization through the quantum scattering process [10, 11]. Third, the thermal fluctuations are important because, for example, the thermal noise power is comparable to the microwave power generated by the magnetization precession [12]. The measurements seem to be satisfactorily treated by the semiclassical theory including micromagnetics. However, the common treatment of angular momentum transfer by the magnet as an immovable scattering potential is shown below by order of magnitude estimate to be inadequate for accurate computation of mean displacement and fluctuations in a nanomagnet. The origin of the stochastic motion arises from the assumption of randomness in either the current or the magnetization. The spin waves seem grafted on rather arising naturally out of the vibration of the spins which constitute the moving magnetization. These points motivate us to question if inclusion of the quantum dynamics of the magnetization may not only provide more accurate connection between the current electrons and the magne- tization but also provide qualitative understanding of the stochastic dynamics as well as bringing out the prospect of coherent motion for precision control. By a quantum theory of the nanomagnet which gives its mean dynamics and fluctuations through scattering between the current electrons and the movable magnet, we hope to illustrate the fundamental aspect of our approach to STT. The development of quantum optics after the laser operation was understood by semiclassical theory provides perhaps an optimistic historical guide for the development of coherent magnetization dynamics after the successes of the semiclassical STT theory.

Conditional Scattering and Stochastic Schrödinger Equation We take the magnetization of a single-domain nanomagnet to be represented by a coherent state of the local spins [13] and view its dynamics in the spinpolarized current as a sequence of scattering by individual electrons. Since each scattering produces a number of outgoing macro-spin states, the serial scatterings create a Monte Carlo trajectory of the macro-spin states which is governed by a stochastic Schödinger equation 14]. Repeated solutions form an ensemble of quantum trajectories for the magnetization from which the mean trajectory and the fluctuations can be obtained. Since the outcomes of the macro-spin states are paired with the scattered spin states of the current electron to form an entangled state, the scattered electron state may be viewed as conditioned on the corresponding macro-spin state. After the first scattering, the electron recedes and the magnetization is left in a reduced density matrix of the outgoing macro-spin states. The second electron from the current may be viewed as the recipient of one of the macro-spin states. This step is equivalent to measurement or decoherence 15], and the magnetization undergoes a Brownian motion and not a quantum random walk. The magnetization noise then has three sources, an intrinsic one due to the uncertainty of the quantum state of the magnetization, two extrinsic ones due to the entangled state after each scattering and due to the particulate nature of each colliding electron with the coherent macro-spin state. The last forms a channel for the mutual effects of the current noise and the magnetization 
noise.

We illustrate the quantum effects with a simple scattering model of the nanomagnet by a regular sequence of electrons and then layer the complexities of the real current [16]. We study here the dynamics of the magnetization as an angular momentum coherent state $|J, \Theta, \Phi\rangle$ [13]. The effect of the spin waves as small deviations from the coherent state is being investigated. $J$ is the quantum number of the total spin $\hat{\mathbf{J}}$ of the localized $d$-electrons in the nanomagnet and is of the order of $10^{6}$ for typical nanomagnets. The angles $\Theta$ and $\Phi$ characterize the direction of the total spin of the nanomagnet. The current electron has the incoming state $|k, \uparrow\rangle$, its wave vector $k$ impinging normally on a ferromagnet film in the $x=0$ plane and its spin along the $z$-axis. The scattering potential, $\delta(x)\left[\lambda_{0} \hat{\jmath}_{0}+\lambda \hat{\mathbf{s}} \cdot \hat{\mathbf{J}}\right]$, is a potential of fixed position but includes a spin independent term indicated by the identity operator $\hat{J}_{0}$ of the total spin state space and the exchange term between the current electron spin and the total local spin. The $\delta$-potential models the FM film of thickness of several nanometer, and the parameters $\lambda_{0}$ and $\lambda$ are the interaction strength between the current electron and one $d$-electron, estimated from two simple exchange-split potentials.

After a scattering event by one current electron, the total state of the system of the nanomagnet and the current electron is entangled [16]. In the Berger [2] basis states $\{| \pm k, \pm\rangle\}$ for the current electron, in which the $z$ axis is rotated in the $z$-J plane to the magnetization direction before the scattering, the conditional macro-spin states associated with the outgoing single electron states are coherent states with an error of the order $1 / J^{2}$,

$$
|\Psi\rangle=\sum_{i=1}^{4} g_{i}\left|J, \Theta_{i}, \Phi_{i}\right\rangle\left|k_{i}, \sigma_{i}\right\rangle,
$$

where $\operatorname{Span}\left(\left|k_{i}, \sigma_{i}\right\rangle\right)=(|-k,+\rangle,|k,+\rangle,|-k,-\rangle,|k,-\rangle)$. The Berger basis is therefore preferable to the original spin basis of the current electron of spin up and down along the $z$-axis, and only the incoming electron state is shown in the original basis. The Berger basis may be viewed as constituting a measurement basis for the current electron with the outcomes associated with the probabilities $G_{i}=\left|g_{i}\right|^{2}, i=1-4$. A conditional state of the macro-spin state of the magnetization is determined by the scattered current electron state, as shown by the Feynman diagrams in Fig. 1. Note that, after scattering, the conditional macro-spin states associated with the electron states $| \pm k,-\rangle$ suffer only a phase change because the flip-flop terms $\widehat{s}_{+} \widehat{J}_{-}+\widehat{s}_{-} \widehat{J}_{+}$in the Heisenberg exchange interaction do not connect the state $|k,+\rangle|J, J\rangle$ to $|k,-\rangle|J, m\rangle$ for any $m<J$.

The evolution of the quantum state of magnetization from $|J, \Theta, \Phi\rangle$ to $\left|J, \Theta_{i}, \Phi_{i}\right\rangle$ stochastically by one spinpolarized current electron may be represented by a rotation, $\left|J, \Theta^{\prime}, \Phi^{\prime}\right\rangle=e^{-i \hat{\mathbf{n}} \cdot \hat{J} \vartheta}|J, \Theta, \Phi\rangle$, with the rotation

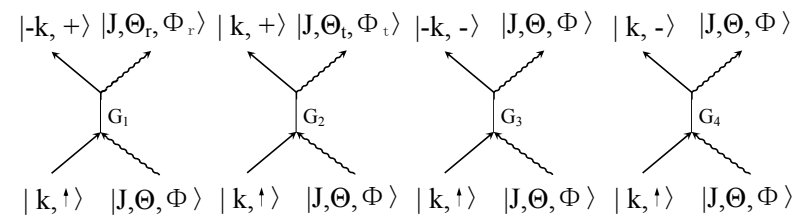

FIG. 1. Four possible states of the nanomagnet in the Berger basis $\{| \pm k, \pm\rangle\}$ after scattering. The straight lines represent the spin-polarized electron and the wavy lines represent the nanomagnet states. The macro-spin state $\left|J, \Theta_{i}, \Phi_{i}\right\rangle$ with suffices $i=1-4$ define the association with the electron spin states in the Berger basis. $G_{i}$ are the conditional probabilities.

axis $\hat{\mathbf{n}}=(-\sin \varphi, \cos \varphi, 0)$ and the rotation angle $\vartheta$ as the random variables with probability outcomes determined by Eq. (11). Since the angle $\vartheta$ is of the order of $1 / J$ and the time interval $\tau$ between two successive electrons is small compared with the time scale of magnetization dynamics, the stochastic rotation of the nanomagnet may be treated as a continuous rotation governed by the stochastic Schrödinger equation,

$$
i \frac{\partial}{\partial t}|J, \Theta, \Phi\rangle=\omega \hat{\mathbf{n}} \cdot \hat{\mathbf{J}}|J, \Theta, \Phi\rangle,
$$

under the effective magnetic field due to the polarized current electrons, producing precession frequency, $\omega \equiv$ $\vartheta / \tau$ about the axis $\hat{\mathbf{n}}$.

Dynamics and Fluctuation of Magnetization, Electric Current and Current Noise To study the switching behavior of nanomagnet at zero magnetic field, we computed an ensemble of 500 runs of Monte Carlo steps, each with sequential scatterings by $N_{e}=1.5 \times 10^{7}$ electrons with the macro-spin $J=10^{6}$. To allow for the three dimension nature of the electron wavevector distribution in a Fermi sphere of radius $k_{F}$ in the normal metal model, the normal component to the interface $k$ obeys the distribution function $f(k)=2 k / k_{F}^{2}$ for $k \in\left[0, k_{F}\right][16]$. In our simulation, the wavevector $k$ of each injected electron is generated randomly according to $f(k)$. The scattered state in each step is randomly selected according to the probabilities $G_{i}$, associated with the macro-spin state $\left|J, \Theta_{i}, \Phi_{i}\right\rangle$ and output electron state $\left|k_{i}, \sigma_{i}\right\rangle$. The effect of STT comes from the random encounter by each incoming electron of a choice of the macro-spin states left by the previous electron, giving rise of a random walk of the magnetization. Fig. 2 shows the direction of the magnetization $\mathrm{J}$ averaged over the ensemble of trajectories and its uncertainties in the Cartesian components $\Delta \mathrm{J}_{\alpha} .500$ runs were found to be sufficient to stabilize the ratio of the uncertainties to the average.

Fig. 2(a) shows that the switching motion also contains a precession about the spin polarization direction of the injected electrons, which reproduced the semiclassical results of being driven by $\mathbf{M} \times \mathbf{S}$ and $\mathbf{M} \times(\mathbf{M} \times \mathbf{S})$ for the magnetization $\mathbf{M}$ and current spin polarization $\mathbf{S}$. 

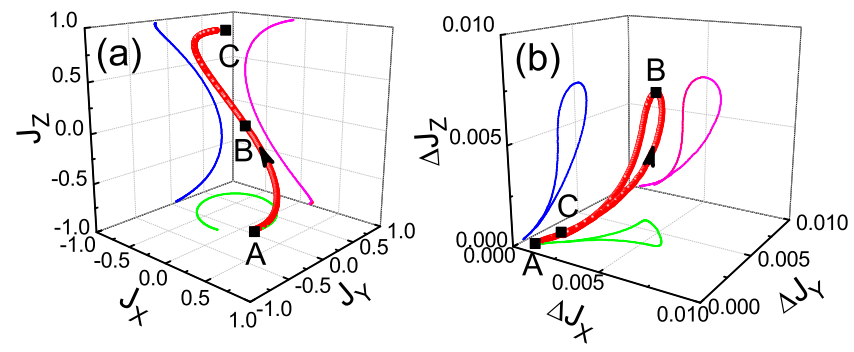

FIG. 2. (color online). Time evolution of magnetization and its noise. (a) The average trajectory of the macro-spin state under STT and its projections onto the Cartesian planes. The Cartesian components $\mathrm{J}_{\alpha}, \alpha=x, y, z$ are normalized in units of $J$. (b) The time evolution of the uncertainties of the macro-spin components and its projections onto the Cartesian planes. $\Delta \mathrm{J}_{\alpha}$ are normalized in units of $J$. The current electron number is $N_{e}=1.5 \times 10^{7}$ and the macro-spin $J=10^{6}$. The initial orientation is $\left(\Theta_{0}, \Phi_{0}\right)=(3,0.5) \mathrm{rad}$. The electron wave vector obey the probability function $f(k)=2 k / k_{F}^{2}$ for $k \in\left[0, k_{F}\right]$ with $k_{F}=13.6 \mathrm{~nm}^{-1}$, and the spin polarization vector is taken $\mathbf{S}=(0,0,1) . \quad \lambda_{0}$ and $\lambda$ are determined by the spin-dependent potential $\Delta_{+}=1.3 \mathrm{~V}, \Delta_{-}=0.1 \mathrm{~V}$, and layer thickness $d=3 \mathrm{~nm}$. The switching time between points $A$ and $C$ is estimated as $1.3 \mathrm{~ns}$ for bias $V=1 \mathrm{mV}$ and cross-section area $\mathcal{A}=10^{4} \mathrm{~nm}^{2}$, by Eq. (3).

It is also found that the number of electrons needed for switching is of the order of $J$. For typical experiments [17], the electric current is of the order of $\mathrm{mA}$ and the switching time is of the order of ns, both consistent with the electron number $N_{e}$ we used. Since the input parameters to our model, namely the Heisenberg exchange energy and the current, are of the order of the physical properties of real systems, we see the ball-park agreement of the switching time as an encouraging sign for the quantum approach so that the noise effects from the theory may be worth being included with the thermal noise in interpretation of experimental measurements. Without the thermal noise, the fluctuations $\Delta \mathrm{J}_{\alpha}$, shown in Fig. 2(b) in time order from point $A$ to $C$, first increase till the midpoint $B$ and then decrease to $C$. The initial state of nanomagnet is a pure angular momentum coherent state $|J, \Theta, \Phi\rangle$ with only intrinsic magnetization noise $\left(\Delta \mathrm{J}_{\alpha} \sim \sqrt{J}\right)$. Scattering by current electrons leads to stochastic motion which accounts for the rise of the extrinsic magnetization noise. As all the trajectories converge to the state $|J, 0,0\rangle$, the magnetization noise finally decreases to the intrinsic quantum fluctuation.

The injected current electron noise can come from either its wave vector distribution $f(k)$ or the uncertainty of its spin state. To identify their effects on the magnetization dynamics of nanomagnet, we include them separately in the simulations and compared the case with no injected electric current noise. As shown in Fig. 3, the inhomogeneous effect of $k$ has less effect on the timeevolution of average value $\langle\Theta\rangle$ and $\langle\Phi\rangle$ and their fluctuations $\Delta \Theta$ and $\Delta \Phi$; while the electron spin noise can
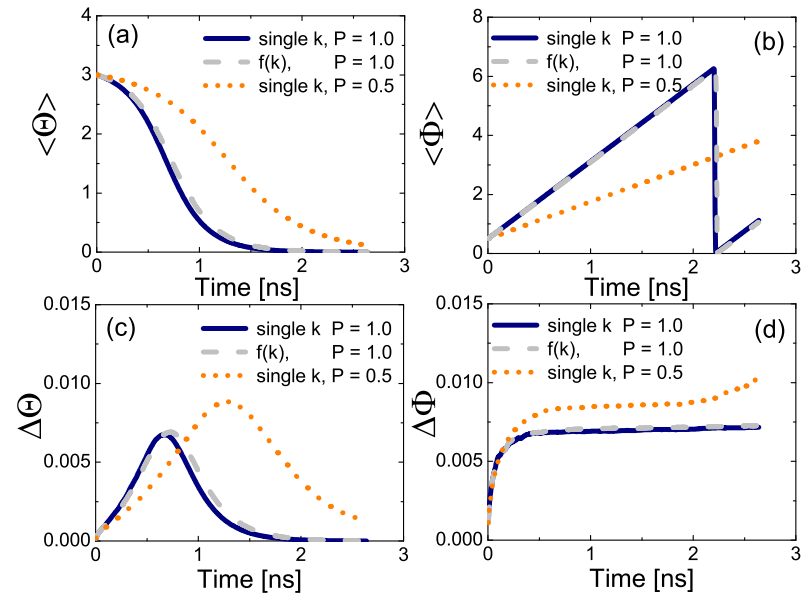

FIG. 3. (color online). Effects of injected current electron noise on the magnetization dynamics of nanomagnet. (a) Time evolution of the average value of $\Theta$. (b) Time evolution of the average value of $\Phi$. (c) Time evolution of the fluctuation of $\Theta$. (d) Time evolution of the fluctuation of $\Phi$. Three cases are considered here: the fully polarized electrons with a single wave vector $2 k_{F} / 3$ (solid line); the fully polarized electrons obey wave vector distribution function $f(k)=2 k / k_{F}^{2}$ for $k \in\left[0, k_{F}\right]$ (dash line); partially polarized electrons with a single wave vector $2 k_{F} / 3$ (dotted line). The spin polarized vector is $\mathbf{S}=(0,0, P)$. The current electron number is $N_{e}=3.0 \times 10^{7}$, and the other simulation parameters are the same as in Fig. 2

change the magnetization dynamics significantly. The magnetization switch time for spin polarization vector $\mathbf{S}=(0,0,0.5)$ is about twice of that for the fullypolarized electrons. The magnetization noise is also obviously enhanced, although its main contribution is still from the scattering process shown in Fig. 1.

The electric current $I$ and current shot noise $S$ in a nanomagnetic junction are given as 18]

$$
I=\frac{e^{2}}{2 \pi \hbar} V \sum_{n=1}^{N_{c}} T_{n}, S=2 e \frac{e^{2}}{2 \pi \hbar} V \sum_{n=1}^{N_{c}} T_{n}\left(1-T_{n}\right),
$$

where $T_{n}$ is the transmission probability of the electron with mixed spin states reduced from the entangled state of Eq. (11). $N_{c}$ is the number of transport channels, estimated as $N_{c} \simeq \mathcal{A} k_{F}^{2}$. The channel sums can be replaced, respectively, by the ensemble average $N_{c} \overline{\delta_{\mu}}$ and variance $N_{c}\left(\Delta \delta_{\mu}\right)^{2}$ of the current electrons, where $\delta_{\mu}$ is a random variable given the value 1 or 0 for a transmitted or reflected electron. By Eq. (3), the time interval $\tau$ between two successive injected electrons is given in terms of the current as $\tau=2 \pi \hbar / N_{c} e V$. Then the number of scattering events in the simulations can be converted to the time scale of magnetization switch. Fig. 4 shows the calculated electron current $I$ and current noise $S$ associated with the magnetization dynamics in Fig. 3. The electric current decreases during the magnetization switch, because the electrons are scattered by spin-dependent potential bar- 

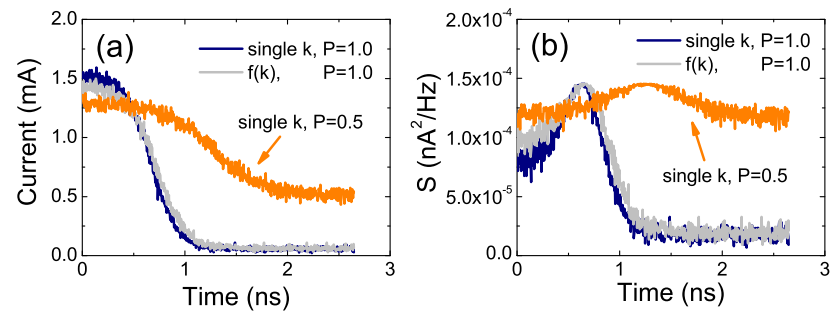

FIG. 4. (color online). Output electric current and current noise. (a) Current during the switching of magnetization in Fig. 3 (b) Current shot noise during the switching of magnetization in Fig. 3

riers of the nanomagnet, $\Delta_{-}=0.1 \mathrm{~V}$ and $\Delta_{+}=1.3 \mathrm{~V}$, at the starting point and final point respectively. The calculated current shot noise $S$ rise and drop in sequence, with the maximum amplitude corresponding to the transmission probability $T=0.5$ for electrons. The calculated current amplitude and switching time are comparable to the experiment results [17]. The amplitude of shot noise of the order of $\mathrm{nA}^{2} / \mathrm{Hz}$ is also consistent with the noise measurement [19]. We found that the change of current and current noise during the magnetization switch decrease with the reduction of current electron spin polarization.

The quantum noise generated in magnetization and electric current due to the scattering can be veiled by the thermal noise in the system at high temperature. The current shot noise will become important when $e V>\kappa \mathcal{T}[18,19]$, where $\kappa$ is the Boltzmann constant and $\mathcal{T}$ is the temperature. For the bias $V=1 \mathrm{mV}$, this gives $\mathcal{T}<11 \mathrm{~K}$, and the shot noise was observed experimentally at $12 \mathrm{~K}[19]$. The magnetization thermal noise can be characterized by the correlator $\left\langle h(t) h\left(t^{\prime}\right)\right\rangle=\left(2 \alpha_{0} \kappa \mathcal{T} / \gamma \mathbf{M} \mathcal{V}\right) \delta\left(t-t^{\prime}\right)$ for the random effective field $h(t)[10,11,20]$, where $\alpha_{0}$ is the Gilbert damping coefficient, $\gamma$ is the gyromagnetic ratio, $\mathbf{M}$ is the magnetization, and $\mathcal{V}$ is the volume for the nanomagnet. Then the thermal fluctuation for the rotation angle $\vartheta(t)=\gamma \int_{t}^{t+\tau} h\left(t^{\prime}\right) d t^{\prime}$ in the time interval $\tau$ will be $\left\langle\delta \vartheta^{2}\right\rangle_{\text {therm }}=2 \alpha_{0} \kappa \mathcal{T} \tau / J \hbar$, here we used the relation $\gamma J \hbar=\mathbf{M} \mathcal{V}$. On the other hand, the quantum noise based on Eq. (2) gives $\left\langle\delta \vartheta^{2}\right\rangle_{\text {quant }} \sim \mathcal{O}\left(1 / J^{2}\right)$. Thus the characteristic temperature that the quantum noise will be comparable to the thermal noise can be roughly estimated by the relation

$$
\alpha_{0} \kappa \mathcal{T} \sim \hbar \omega_{s}
$$

where $\omega_{s}=1 / J \tau$ is a characteristic frequency related to the switch time of the nanomagnet. For the nanomagnet in Ref. [17], $\alpha_{0} \sim 0.025$, together with our simulation results above $J \tau \sim 0.1 \mathrm{~ns}$, the characteristic temperature $\mathcal{T}$ is about $3 K$. Relation (4) shows that the quantum magnetization noise will be more important for smaller Gilbert damping coefficient, smaller nanomagnet, or shorter magnetization switch time.

Conclusion The quantum trajectory method we have developed not only reproduced the semiclassical results of STT, but also revealed the generation of quantum noise and the noise transfer between the nanomagnet and current. More quantum phenomena in the STT-related physics are anticipated in further studies based on this quantum picture.

Acknowledgement We acknowledge support of this work by the U. S. Army Research Office under contract number ARO-MURI W911NF-08-2-0032 and thank W. Yang, Y.J. Zhang, H. Suhl, and I.N. Krivorotov for valuable discussions.

* lsham@ucsd.edu

[1] J.C. Slonczewski, J. Magn. Magn. Mater. 159, L1(1996).

[2] L. Berger, Phys. Rev. B 54, 9353 (1996).

[3] D.C. Ralph and M.D. Stiles, J. Magn. Magn. Mater. 320, 1190 (2008).

[4] T. Devolder, J. Hayakawa, K. Ito, H. Takahashi, S. Ikeda, P. Crozat, N. Zerounian, J.-V. Kim, C. Chappert, and H. Ohno, Phys. Rev. Lett., 100, 057206 (2008).

[5] H. Tomita, K. Konishi, T. Nozaki, H. Kubota, A. Fukushima, K. Yakushiji, S. Yuasa, Y. Nakatani, T. Shinjo, M. Shiraishi, and Y. Suzuki, Applied Physics Express 1, 061303 (2008).

[6] Y.-T. Cui, G. Finocchio, C. Wang, J.A. Katine, R.A. Buhrman, and D.C. Ralph, Phys. Rev. Lett., 104, 097201 (2010).

[7] S. Garzon, L. Ye, R.A. Webb, T.M. Crawford, M. Covington, and S. Kaka, Phys. Rev. B 78, 180401 (2008).

[8] X. Cheng, C.T. Boone, J. Zhu, and I.N. Krivorotov, Phys. Rev. Lett. 105, 047202 (2010).

[9] H. Suhl, Relaxation processes in micromagnetics (Oxford University Press, 2007).

[10] J. Foros, A. Brataas, G. E. W. Bauer, and Y. Tserkovnyak, Phys. Rev. B 79214407 (2009).

[11] A. L. Chudnovskiy, J. Swiebodzinski, and A. Kamenev, Phys. Rev. Lett. 101, 066601 (2008).

[12] A. Slavin and V. Tiberkevich, IEEE Transactions on Magnetics 45, 1875 (2009).

[13] W.-M. Zhang, D.H. Feng, and R. Gilmore, Rev. Mod. Phys. 62, 867 (1990).

[14] H. J. Carmichael, An open systems approach to quantum optics (Springer, Berlin, 1993).

[15] H. M. Wiseman and G. J. Milburn, Quantum Measurement and control (Cambridge University Press, Cambridge, 2010).

[16] See supplementary material for details.

[17] I.N. Krivorotov, N.C. Emley, J.C. Sankey, S.I. Kiselev, D.C. Ralph, and R.A. Buhrman, Science, 307, 228 (2005).

[18] Ya.M. Blanter and M. Büttiker, Phys. Rep. 336, 1 (2000).

[19] K. Sekiguchi, T. Arakawa, Y. Yamauchi, K. Chida, M. Yamada, H. Takahashi, D. Chiba, K. Kobayashi, and T. Ono, Appl. Phys. Lett. 96, 252504 (2010).

[20] W.F. Brown, Phys. Rev. 130, 1677 (1963). 